Indonesian Journal of Chemical

Science and Technology

State University of Medan

$e-I S S N$ : 2622-4968, p-ISSN : 2622-1349

IJCST-UNIMED, Vol. 02, No. 1, Page; 80-83

Received: Desember 10, 2018 Accepted : Januari 22, 2018 Web Publised; January 28, 2019

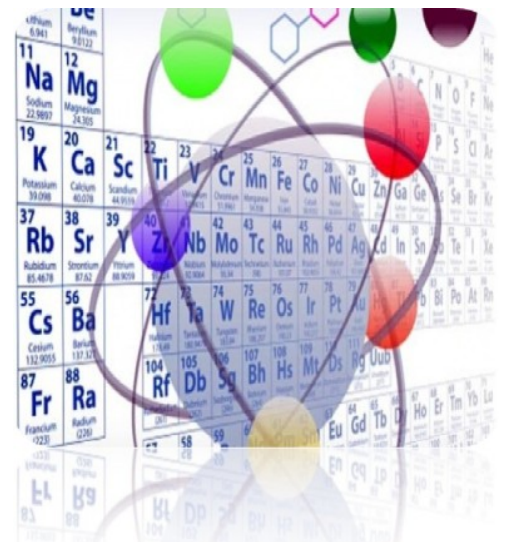

\title{
Derivatisation of 2,4 (dinitrophenyl hydrazine) DNPH in Canola oil oxidation
}

\author{
Marini Damanik ${ }^{a *}$, Michael Murkovic ${ }^{b}$ \\ a State University of Medan, Address, Medan 2021, Indonesia \\ ${ }^{b}$ TU-Graz, Address, Graz 8010,Austria \\ *Email : marini@unimed.ac.id
}

\begin{abstract}
Lipid oxidation during heating started at the allylic position of the double bond of unsaturated fatty acids. Primary lipid oxidation which was produced during heating are generally unstable and directly decompose into secondary oxidation products. The oxidative and thermal stability of Rapeseed oil was evaluated using Rancimat 679. Oil samples were heated at $120^{\circ} \mathrm{C}$ for 10 hours which are taken started from 5, 6, 6.5, 7, 7.5, 8, 9 and 10 hours. Derivatization with DNPH eluted with a mixture of methanol (60\%) and water (40\%). The aldehydes are analysed by High Performance Liquid Chromatography-Diode Array Detection (HPLC-DAD) after derivatisation with DNPH and identified by high-resolution MS using an orbitrap-based mass spectrometer. HPLC is the suitable method to investigate the non-volatile aldehydes formed by decomposition of hydroperoxides. The result of this oxidation process were described that the maximum point of the oil oxidation was at 9 hours which peak area 378. For oxidation process at the 10 hours the area peak is decreased which point 344
\end{abstract}

Keywords: lipid oxidation

\section{Introduction}

Canola oil is considered to be a nutrionatilly well-balanced because it has a low content of SFA, a high content of MUFA, and a 2: 1 ratio of $\omega 6$ and $\omega 3$ PUFA. It has a high content of $\alpha$-linolenic acid (8-12\%) compared to other vegetables oils such as soybean $(8 \%)$, sunflower $(0.2 \%)$, olive $(0.8 \%)$ and corn $(0.7 \%)$ From information stated above, the high polyunsaturated fats make it easily to rancid if it's heating in the high temperature. ${ }^{1}$ Study on the oxidative stability of canola oil is needed in order to measure the contain of oxidation product. This reaction leads to the formation of intermediate compounds which named is hydroperoxide as a primary oxidation product. Because of unstable, these products will be change fastly to the secondary product oxidation including carbonyl compound. ${ }^{1}$ Carbonyl compound is more stable than peroxides and it always used for evaluating the quality of oil ${ }^{2,3,4}$

The content of primary (PO) and secondary (SO) oxidation products of canola oil was measured. Peroxide value showed the highest contribution $(53,69 \%)$ to the $\mathrm{PO}$, whereas the $\mathrm{SO}$ was much less significant $(0,36 \%)$. However, acid value contribution to the production of hydroperoxides and carbonyls was $12.75 \%$ and $29.82 \%$, respectively. Polyene index showed a relatively low contribution to the PO $(6.71 \%)$ but contributed highly to the SO (21.82\%). Carbonyl compounds are more stable than hydroperoxides 
and their measurements is a good index for oxidative changes in lipids. ${ }^{1}$

Several methods has been conducted on derivatizing carbonyls. The most widely used derivatization agent is 2,4 dinitrophenylhydrazine (DNPH). Carbonyl compounds, in particular aldehydes, are reactive volatile substances. 2,4Dinitrophenylhydrazine can be used to qualitatively detect the carbonyl functionality of a ketone or aldehyde functional group. Measuring the color intensity of hydrazones as a product reaction can be an estimation value of rancidity. The positive test of signaled which produce the different colour such as an aromatic with red precipitate and aliphatic with a more yellow color.

Aldehydes and ketones which was produced from frying fats are reacted with 2,4dinitrophenylhydrazine in acidic solution for $1 \mathrm{~h}$ at room temperature. It was $\mathrm{s}$ simple, fast and a selective determination of higher carbonyl group. Using a reversed phase HPLC column that is eluted with a very steep gradient between methanol and tert-butylmethyl ether (TBME) with wavelength set at $370 \mathrm{~nm}$. This method resulted as a miliequivalents of carbonyls per $\mathrm{kg}$ of fat which was produced from oxidative cleavage of double bonds in unsaturated fatty acids. ${ }^{5}$

In this research, the formation of aldehyd as a secondary product oxidation reacted with 2,4 DNPH and then to separate the derivatives of the target analytes. Started with the derivatives of short-chain aldhydes to identify and quantity the aldehyd and keton compound, the excess of reagent, and other compounds complex mixture of carbonyls to be expected and the problem of separating all the individual components, we instead tried to find suitable HPLC conditions that would result in only one peak, easily integrated for the sum of all analytes.

\section{Experimental}

\subsection{Chemical and Instrumentation}

DNPH was purchased from Sigma-Aldrich (Vienna, Austria), hydrochloric acid ( $\mathrm{HCl} 37 \%$ ) was purchased from Merck (Damstadt, Germany), all solvents (e.g. methanol, ACN, aceton) used were HPLC grade were purchased from Merck (Damstad, Germany), acetic acid was purchased from Roth (Karlsruhe, Germany), Canola (liquid at room temperature), oils were purchased from a local supermarket in Graz, Austria.

\subsection{Procedure Oxidation of Canola Oil}

Canola oil (10 g) were heated in 679 Rancimat at $120^{\circ} \mathrm{C}$ during $10 \mathrm{~h}$ which are taken started from $5,6,6.5,7,7.5,8,9$ and 10 hours for oil sample analysis.

\section{Derivatisation with DNPH}

Oil sample is dissolved in $1 \mathrm{ml}$ acetonitrile. The filtered extract which is the upper layer derivatized to $900 \mu \mathrm{L}$ of the reagent DNPH. The mixture kept in the dark place at room temperature for at least 1 h. Derivatization with DNPH eluted with a mixture of methanol $(60 \%)$, water $(40 \%)$ and $0,1 \%$ formic acid which gives a better selectivity due to the absorption maximum of diode array detector DADHPLC at $400 \mathrm{~nm}$. A suitable aliquot injected into the high-performance liquid chromatograph equipped with a quartenary pump, vacuum degasser, autosampler, and variable wavelenght detector (DAD Detector). The colum was an RP LichrocartPurosphere Star 100 (55 x 2 mm, $3 \mu \mathrm{m})$.

\section{Result and discussions}

\subsection{Characterisation Analysis}

Oxidation of edible oils occurs when MUFA and PUFA are mainly glycerol bound, react with atmospheric $\mathrm{O}_{2}$. Primary oxidation products, hydroperoxides, are formed through different chemical mechanisms. Even though fresh vegetable oils were not oxidized extensively, heating altered the content of lipid oxidation products considerably. Hydroperoxides are unstable to heat and will easily decompose to the more stable secondary oxidation products under heating. The decomposition of hydroperoxides was probably faster than the formation.

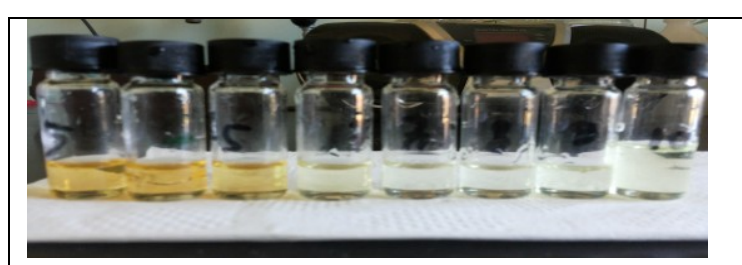

Figure 1. The colour result of oxidation process from $5 \mathrm{~h}$ to $10 \mathrm{~h}$ by Rancimat 679

The oxidation process with Rancimat 679 of canola oil was a good analytical index to measure oxidation alteration. The oil was heated at $120{ }^{\circ} \mathrm{C}$ for $10 \mathrm{~h}$ in the formation of primary and secondary 
oxidation. At the started time for taking oil in $5 \mathrm{~h}$ of oxidation, the oil is yellow, then finally becoming pale yellow to white in a liquid form at $10 \mathrm{~h}$ for oxidation time.

The result of this oxidation process were described that the maximum point of the oil oxidation was at 9 hours which peak area 378 . Based on the oxidation theory, propagation step of oil oxidation started which means the fatty acid reacts readily with molecular oxygen, thereby creating a peroxyl-fatty acid radical. This radical is also an unstable species that reacts with another free fatty acid, producing a different fatty acid radical and a lipid peroxide, or a cyclic peroxide if it had reacted with itself. This cycle continues, as the new fatty acid radical reacts in the same way. For oxidation process at the 10 hours the area peak is decreased which point 344 (Table 1).

Table 1. Oxidation of Canola oil's peak areas.time

\begin{tabular}{|l|l|l|l|l|l|l|}
\hline Time (h) & 6 & 7 & 7,5 & 8 & 9 & 10 \\
\hline $\begin{array}{l}\text { Peak } \\
\text { area }\end{array}$ & 126 & 206 & 274 & 361 & 378 & 344 \\
\hline
\end{tabular}

In this time the radicals react with the non radicals and it always produce the other radical. The term of this reaction called chain reaction mechanism. Thus, the reaction will be stop when two radicals react to produce a non radical species. One important point of this research is the using of 2,4 DNPH to catch of the aldehyd product. From the chromatogram display showed that the peak of $2,4 \mathrm{DNPH}$ reduced if the time of oxidation process is long because the end products of lipid peroxidation are reactive aldehydes.

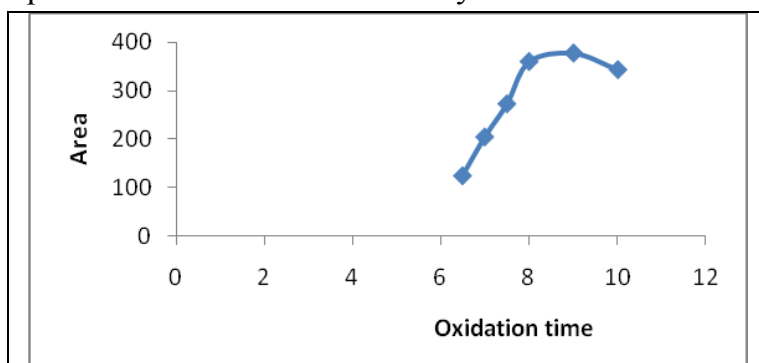

Figure 2. The profile of oxidation value because of the increasing of temperature

For some of retention time which are produced, we assumed that the area will be reduced during the oxidation time. The fourth points of selected peaks which are 10.04, 11.40, 14.48 and 15.84 showed that in the $9 \mathrm{~h}$ produced the highest value of detector area and it would be decreased significantly at the $10 \mathrm{~h}$ (Table 2).

Table 2. Selected peak which are representing the kinds of oxidation product.

\begin{tabular}{|l|l|l|l|l|}
\hline \multirow{2}{*}{$\begin{array}{l}\text { Oxidation } \\
\text { time (h) }\end{array}$} & \multicolumn{4}{|c|}{ Selected peak } \\
\cline { 2 - 5 } & 10,04 & 11,40 & 14,48 & 15,84 \\
\hline 6,5 & 1,03 & 10,01 & 4,40 & 0,3 \\
\hline 7 & 13,93 & 33,83 & 11,80 & 9,39 \\
\hline 7,5 & 15,26 & 53,31 & 17,48 & 17,13 \\
\hline 8 & 27,04 & 95,05 & 26,62 & 26,78 \\
\hline 9 & 32,15 & 102,38 & 30,39 & 34,33 \\
\hline 10 & 22,70 & 68,42 & 16,39 & 19,95 \\
\hline
\end{tabular}

Oxidation of edible oils occurs when MUFA and PUFA, which are mainly glycerol based, react with atmospheric $\mathrm{O}_{2}$. Hydroperoxides are unstable to heat and will easily decompose to the more stable secondary oxidation products under such conditions. The decomposition of hydroperoxides was probably faster than the formation.

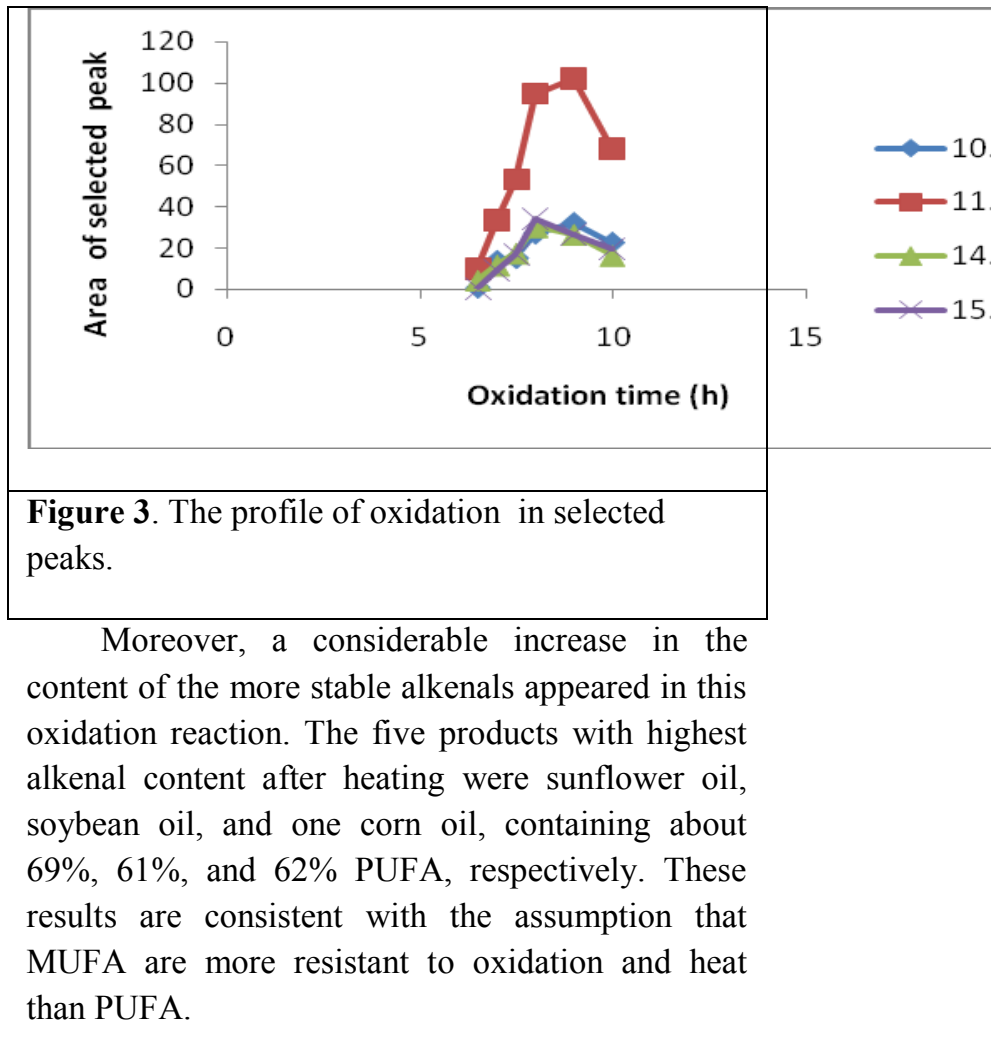




\section{Conclusion}

The formation of carbonyls was quantified during oxidation of canola by derivatization of the formed products with DNPH which is a selective reagent for aldehydes and ketones. Some of the oxidized fragments of canolawere identified by LC-MS/MS - using APCI in negative modebeing. The fourth points of selected peaks which are $10.04,11.40,14.48$ and 15.84 showed that in the $9 \mathrm{~h}$ produced the highest value of detector area and it would be decreased significantly at the $10 \mathrm{~h}$. Using a Rancimat for reproducible oxidation experiments with a constant air flow at defined temperatures $\left(120^{\circ} \mathrm{C}\right)$ the production of carbonyls showed a good repeatability. The formation of carbonyls from triolein showed a maximum after $9 \mathrm{~h}$ with a slight or no decrease during prolonged oxidation.

\section{References}

1. Farhoosh, R., Samaneh, P (2009). Relative contribution of compotional parameter to the primary and secondary oxidation of canola oil. Food Chemistry 114, pp. 1002-1006

2. Woyewoda, A., Shaw S., Ke P., Burns B., (1986). Recommended laboratory methods for assessment of fish quality, numeral 1448: canadian technical report of fisheries and aquatic sciences. Minister of Supply and Services Canada, Halifax, Canada, pp. 143

3. R. Farhoosh R., \& Moosavi, S.M.R (2008). Carbonyl value in monitoring of the quality of used frying oils. Analytica Chimica Acta, 617, $18-21$

4. Yasushi, E., Chang Mo, L., Endo, M.T., Fujimoto, K., (2001). A modified method for the estimation of total carbonyl compounds in Li heated and frying oils using 2-propanol as a solvent, JAOCS, 78(10, pp. 1021-1024.

5. Schulte, E., (2002). Determination of higher carbonyls compounds in used frying fats by HPLC of DNPH derivatives. Anal Bioanal Chem $372: 644-648$. 